\title{
CD276 Promotes Vasculogenic Mimicry Formation in Hepatocellular Carcinoma via the PI3K/AKT/MMPs Pathway
}

This article was published in the following Dove Press journal: OncoTargets and Therapy

\author{
Rui Cheng (D) ${ }^{1, *}$ \\ Bi Wang',* \\ Xin-ran Cai ${ }^{1}$ \\ Zhi-shan Chen' \\ Qiang Du' \\ Liang-yi Zhou' \\ Jing-min $\mathrm{Ye}^{\mathrm{l}}$ \\ Yan-ling Chen ${ }^{1,2}$
}

'Department of Hepatobiliary Surgery and Fujian Institute of Hepatobiliary Surgery, Fujian Medical University Union Hospital, Fuzhou, Fujian 35000I, People's Republic of China; ${ }^{2}$ Fujian Medical University Cancer Center, Fuzhou, Fujian 35000I, People's Republic of China

*These authors contributed equally to this work
Correspondence: Yan-ling Chen Department of Hepatobiliary Surgery and Fujian Institute of Hepatobiliary Surgery, Fujian Medical University Union Hospital, Xinquan Road 29\#, Fuzhou, Fujian 350001 , People's Republic of China Email chenyanling64@।26.com
Purpose: CD276 protein expression and vasculogenic mimicry (VM) formation are associated with the poor prognosis of hepatocellular carcinoma (HCC) patients. Although both the effects of CD276 and VM formation involve the activation of matrix metalloproteinases, and their relationship has not yet been explored. The following study investigated the effect of CD276 expression on VM formation and the potential mechanisms.

Materials and Methods: CD276 expression and VM were examined in commercial tissue microarrays by immunohistochemistry and CD31/PAS double staining. Tumor cell proliferation, invasion, migration and, tube formation were detected in vitro after transfecting HCC cell lines with an shRNA lentiviral vector against CD276. The expression of MMP14, MMP2, VE-cadherin, E-cadherin, and vimentin and MMPs activation was detected by Western blot, immunofluorescence and gelatin zymography assay. In addition, an orthotopic xenograft model of HCC cells was established in vivo, after which VM was detected, along with its marker molecules.

Results: CD276 expression was associated with VM and poor prognosis in HCC patients. RNA interference of CD276 reduced tumor cell proliferation, invasion, migration, and VM formation in vitro and in vivo. Furthermore, CD276 knockdown up-regulated the expression of E-cadherin but inhibited the phosphorylation of AKT, the expression of MMP14, MMP2, VE-cadherin, vimentin and the activation of MMP2 and MMP9 in HCC cell lines.

Conclusion: CD276 may promote VM formation by activating the PI3K/AKT/MMPs pathway and inducing the EMT process in HCC. CD276 may serve as a promising candidate for the anti-VM treatment of HCC.

Keywords: hepatocellular carcinoma, vasculogenic mimicry, CD276, matrix metalloproteinases

\section{Introduction}

Hepatocellular carcinoma (HCC) is one of the most common primary liver cancers and the third most frequent cause of cancer death worldwide. ${ }^{1}$ Despite the fact that considerable advances have been made in the treatment of malignant tumors, the prognosis of HCC patients remains poor. ${ }^{2}$ The high mortality rate is mainly attributed to vascular invasion and metastasis. ${ }^{3}$ Angiogenesis is crucial for promoting the growth and metastasis of $\mathrm{HCC}^{4}$ Besides angiogenesis, several other mechanisms of tumor vessel formation have also been proposed, including vascular co-option, vascular intussusception, vascular cooption and vasculogenic mimicry (VM). ${ }^{5} \mathrm{VM}$ has been accepted as a new model of neo-vascular supply in aggressive 
tumors. ${ }^{6}$ It refers to the formation of functional blood channels by highly aggressive tumor cells without the involvement of endothelial cells. Previous studies have employed CD34 or CD31 and periodic acid-Schiff (PAS) dual staining to distinguish the matrix-rich morphological pattern of VM in endothelial cells undergoing angiogenesis. ${ }^{7} \mathrm{VM}$ has been found in several cancer types, including $\mathrm{HCC}^{8}{ }^{8}$ Its presence is closely associated with high tumor grade, increased degree of invasiveness, and shorter overall survival in patients. ${ }^{9,10}$ Moreover, VM not only participates in nutrition and oxygen supply necessary for tumor growth but also provides the metastatic routes for tumor cells. ${ }^{11}$ The direct exposure of tumor cells lining the inner surface of VM channels to blood flow indicates an escape route for the metastasis process. In addition, during VM development, tumor cells acquire malignant characteristics such as invasiveness, poor differentiation, mesenchymal phenotype and self-renewability, ${ }^{12}$ which are essential for cancer progression and metastasis. Therefore, an ideal anti-vascular therapeutic agent should target both angiogenesis and VM formation.

CD276 (B7-H3), an important member of the B7 superfamily, is encoded by chromosome 15 in humans. Although CD276 mRNA is widely expressed in normal human tissues, its protein expression is generally limited, suggesting the presence of a critical post-transcriptional control mechanism. ${ }^{13} \mathrm{CD} 276$, which has an important role in innate immunity and $\mathrm{T}$ cell-mediated adaptive immunity ${ }^{14}$ has been shown to be highly expressed in various tumors, including HCC; Its high expression often correlates with negative prognosis and poor clinical outcomes. ${ }^{15-18}$ Similar to other immune checkpoints of the B7-CD28 pathways, CD276 is expressed in immune cells (such as antigen-presenting cells or macrophages), it regulates the function of $\mathrm{T}$ cells as a second signal molecule. ${ }^{19}$ Besides its role in modulating tumor immunity, it also has a non-immunological function in regulating tumor aggressiveness. Accumulating evidence has demonstrated that CD276 is involved in modulating migration and invasion of various cancer cells, including HCC. ${ }^{17,18,20,21} \mathrm{Up}$ to date, however, the molecular mechanisms though which CD276 participates in various tumor-associated biological processes remain elusive and need to be further explored.

Some studies have indicated that CD276 might induce the migratory potential and invasiveness of tumor cells by increasing the expression of metastasis-associated proteins such as matrix metalloproteinases (MMPs). ${ }^{22,23}$ It has also been reported that MMPs are closely associated with VM formation in hepatocellular carcinoma. ${ }^{9,24}$ In our previous study, we also found that the mechanisms of VM formation might involve the up-regulation of expression and activation of MMPs. ${ }^{25}$ Highly aggressive hepatocellular carcinoma cells may secrete matrix metalloproteinases, express vascular endothelial cadherin (VE-cadherin) and laminin to induce extracellular matrix remodeling and promote VM formation. ${ }^{24}$

Although the presence of CD276 and its implications have been shown in HCC, the correlation between CD276 and $\mathrm{VM}$ in $\mathrm{HCC}$ and the underlying mechanisms remain unclear. In this study, we attempted to investigate the effects of CD276 expression on VM formation and the potential mechanisms.

\section{Materials and Methods Tissue Microarrays Analysis}

Two commercial tissue microarrays (TMA) from 93 hepatocellular carcinoma patients were purchased from Shanghai Outdo Biotech (Shanghai, China) with the TMA's accompanying clinical pathology and follow-up data. Briefly, HCC tissue specimens were obtained from the patients who underwent surgery between January 2007 and November 2009. Their HCC diagnosis was confirmed by pathology. The mean age of patients was 54 years (ranging from 25 to 73 years). Tumor histological grade was defined following the World Health Organization classification. The definition of the follow-up period was from the date of surgical resection to death or until September 2013 (range: 1-80 months). Survival time was defined as the period between the date of surgical resection and the date of death or the patient's last follow-up. The use of the tissue microarrays was approved by the Ethics Committee of Shanghai Outdo Biotech (Ethical approval number: T19-0361).

\section{Immunohistochemistry and CD3 I/PAS Double Staining}

Antigen retrieval was performed by heating the tissue sections from TMA in EDTA for 10 min. Sections were cooled down at room temperature and were immersed in $0.3 \% \mathrm{H}_{2} \mathrm{O}_{2}$ solution for 20 min to block endogenous peroxidase activity; Then the sections were incubated overnight at $4{ }^{\circ} \mathrm{C}$ with the primary antibody (Goat polyclonal Anti-CD276 antibody; R\&D System, Minneapolis, USA). Negative controls were treated by replacing the 
primary antibody with PBS, after which the samples were incubated with the secondary antibody at $37{ }^{\circ} \mathrm{C}$ for 30 min. The color was developed using a 3,3'diaminobenzidine chromogen (DAB) solution. Microarrays were separately analyzed by two pathologists blinded to the patients' clinical information. Sections were considered to be positive when tumor cells showed cytoplasmic or membranous CD276 immunostaining. The stained area and the intensity of the stain were independently scored as follows: $\leq 33 \%$ of the cancer cells, $1 ;>33$ to $\leq 66 \%$ of the cancer cells, $2 ;>66 \%$ of the cancer cells, 3 . The intensity of staining was scored as follows: absent/ weak, 1; moderate, 2; strong, 3. Each section had a final grade that was calculated by multiplying the area and intensity scores. For analysis, sections with a final score of $\leq 3$ were defined as tumors with low CD276 expression group (the low-expression group), whereas sections with a final score of $>3$ were defined as tumors with high CD276 expression group (the high-expression group). ${ }^{26}$ For validation, we searched the human protein atlas (http://www.proteinatlas.org/) database to evaluate the differential protein expression levels of CD276 and the Kaplan-Meier plotter (https://kmplot.com/analysis/) database to analyze the prognostic effect of CD276 on patients with HCC.

For the CD31/PAS double staining, immunohistochemical staining with CD31 (Zhongshan Biology Technology Co., Beijing, China) was first performed on the sections from TMA as described above before PAS staining. Then, the sections were counterstained with a periodic acidSchiff (PAS) Kit (Sigma, USA). Finally, all of the sections were counterstained with hematoxylin, dehydrated, and mounted.

\section{Cell Culture and Transfection}

The HCC cell lines (Huh7, SK-HEP-1, and SMMC7721) were obtained from the American Type Culture Collection (Rockville, MD, USA). The HCC cell lines (Bel7402, MHCC97H and Bel7404) were obtained from Cell Bank of Chinese Academy of Sciences (Shanghai, China). SKHEP-1 was cultured in Roswell Park Memorial Institute 1640 supplemented with $10 \%$ fetal bovine serum (Invitrogen, Carlsbad, CA, USA). Other cell lines were cultured in Modified Eagle's Medium supplemented with $10 \%$ fetal bovine serum. Cells were maintained at $37{ }^{\circ} \mathrm{C}$ and $5 \% \mathrm{CO}_{2}$ in a humidified atmosphere. The expression of CD276 in each cell line was evaluated by Western blotting.
Lentiviral vectors encoding the enhanced green fluorescent protein (EGFP) sequence containing shRNA specific to CD276 (LV-shCD276) and a non-targeted control shRNA (LV-NC) were constructed by Shanghai Genechem Co. (Shanghai, China). Lentiviral vectors with LVshCD276 or LV-NC were transfected into HCC cells according to the manufacturer's instructions. Briefly, LVshCD276 and LV-NC, each at a multiplicity of infection (MOI) of 50, were transfected into $2 \times 10^{4}$ cells cultured in 12-well plates with $5 \mu \mathrm{g} / \mathrm{mL}$ of Polybrene. The sequence of the CD276 shRNA used in this study was as follows: 5'caGCTGACAGATACCAAACAG-3'. At 24 hours after the transfection, cells were harvested for analysis. Cells without any treatment were used as blank controls (Control).

\section{Three-Dimensional Culture}

A 96-well plate was placed on ice for pre-cooling and Matrigel (BD, Franklin lake, New Jersey, USA) was thawed at $4^{\circ} \mathrm{C}$ and added to each well of the plate $(30$ $\mu \mathrm{L} /$ well). Then the plate was moved into a $37{ }^{\circ} \mathrm{C}$ incubator containing $5 \% \mathrm{CO}_{2}$ for $12 \mathrm{~h}$ to solidify the Matrigel. Tumor cells suspended in the medium were tiled on the gel and incubated at $37^{\circ} \mathrm{C}$ for $24 \mathrm{~h}$. VM tubes were captured under an inverted microscope. Each experiment was performed in triplicate.

\section{Immunofluorescence Staining}

The transfected cells were plated onto chamber slides and fixed in ice-cold methanol. The primary antibody against VE-Cadherin (Cell Signaling, Danvers, MA, USA) was used at a 1:400 working dilution. Fluorescein isothiocyanate rabbit immunoglobulin $G$ antibody (Santa Cruz Biotechnology, Santa Cruz, CA, USA) was used as a label for the immunofluorescence assay. After immunolabelling, the cells were washed, stained with DAPI (Sigma), mounted and then viewed under a fluorescent microscope (Nikon, Japan).

\section{Cell Proliferation Assay}

To assess cellular proliferation, cell proliferation was detected by the cell counting kit-8 (CCK-8) method. After transfection for $24 \mathrm{~h}$, cells were counted with adjusted cell concentration and 5000 cells per well were seeded in 96-well plates. Cell proliferation was measured using a CCK-8 (Dojindo, Kumamoto, Japan) according to the manufacturer's instructions. Proliferation rates were determined at 24, 48, 72 and 96 h of culture by measuring 
the absorbance at $450 \mathrm{~nm}$ in a microplate reader (Bio-Rad, USA). Triplicate wells were set for each group.

\section{Invasion and Migration Assay}

The cell invasion assay was performed with 24-well Transwell chambers (Corning Costar, Cambridge, MA, USA) with 8 - $\mu \mathrm{m}$ pore polycarbonate membranes precoated with a layer of Matrigel (BD, Franklin lake, New Jersey, USA). The transfected cells suspended into $100 \mu \mathrm{L}$ medium were seeded in the upper chamber $\left(4 \times 10^{4}\right.$ cells/ well). The lower chamber contained $500 \mathrm{~mL}$ medium with $10 \%$ FBS as chemotaxin. After 48 hours of incubation at $37^{\circ} \mathrm{C}$, non-invaded cells on the top of the membrane were removed. The invaded cells were washed with PBS, fixed with $4 \%$ paraformaldehyde and stained with $0.1 \%$ crystal violet. The number of cells on the membrane of the lower chamber was counted by light microscopy. The migration assay was also performed with Transwell chambers and wound-healing assay. For the wound-healing assay, the cells were seeded in 6-well plates and were allowed to grow until they reached a $90 \%$ concentration. When the cells formed a monolayer, a straight scratch was made in the center of each well using a micropipette tip, and the cells were washed with PBS and incubated in a serum-free medium. Microphotographs were taken and the migration rate (MR) was monitored at $48 \mathrm{~h}$. The following formula was used to calculate MR: MR $=\left(d-d^{\prime}\right) / d$, where $d$ is the length of the wound at time 0 , and $\mathrm{d}^{\prime}$ is the length at 48 h. Each experiment was performed in triplicate.

\section{Western Blotting and Gelatin Zymography Assay}

We used the online database Gene Expression Profiling Interactive Analysis (GEPIA, http://gepia.cancerpku.cn/ index.html), ${ }^{27}$ an interactive web server based on The Cancer Genome Atlas (TCGA), to explore the correlation between CD276 and key molecules of PI3K/AKT/MMPs pathway.

The expressions of AKT, phosphorylated AKT (pAKT), MMP14, MMP2, VE-cadherin, E-cadherin, vimentin in cell lines were detected by Western blotting as verification. LY294002, a PI3K inhibitor, was purchased from Cell Signaling Technology (Danvers, MA, USA) and added to the cell culture medium at a final concentration of $20 \mu \mathrm{mol} / \mathrm{L}$ as a positive control. HCC cells were washed with PBS twice and lysed on ice with a RIPA lysis buffer $(1 \mathrm{~mL})$ containing protease and phosphatase inhibitor for $30 \mathrm{~min}$. Cell lysate protein content was determined with a Bicinchoninic acid (BCA) protein assay kit. An equivalent amount of whole protein extracts was separated by $10 \%$ SDS-PAGE under denaturing conditions and then transferred to polyvinylidene difluoride (PVDF) membranes (Millipore, Billerica, MA, USA). Membranes were then blocked with $5 \%$ non-fat milk powder in Tris-buffered saline containing $0.1 \%$ Tween 20 (TBST) and probed with primary antibodies at $4^{\circ} \mathrm{C}$ overnight. After washing with TBST, the membranes were incubated with secondary antibodies. Bands were visualized with an ECL detection kit (Pierce, USA), and normalization was made against GAPDH expression. The intensities of the bands were quantified by Image $\mathrm{J} \underline{\text { soft }}$ ware. Antibodies for research were as follows: CD276 (R\&D System, Minneapolis, USA); AKT, pAKT, MMP14, MMP2, VE-cadherin, E-cadherin, vimentin, GAPDH (Cell Signaling, Danvers, MA, USA).

Additionally, the activity of MMP-9 and MMP-2 was measured with a commercial gelatin zymography kit (Xin Fan Biology Technology Co., Nanjing, China) according to manufacturer's instructions.

\section{Orthotopic Transplant Mouse Model of HCC Cells}

To examine the effects of CD276 on tumor growth and VM formation in vivo, an orthotopic transplantation tumor model with HCC cells was established. Balb/c male nude mice, 4-5 weeks old, weighing $20-25 \mathrm{~g}$, were obtained from Slac Laboratory Animal Co. Ltd. (Shanghai, China). All the animals were housed in an environment with a temperature of $22 \pm 1{ }^{\circ} \mathrm{C}$, relative humidity of $50 \pm$ $1 \%$, and a light/dark cycle of $12 / 12 \mathrm{hr}$. All animal studies (including the mice euthanasia procedure) were done in compliance with the regulations and guidelines of Fujian Medical University (Fuzhou, China) institutional animal care and conducted according to the AAALAC and the IACUC guidelines.

Bel7404 cells transfected with LV-shCD276 or LV-NC control were harvested and resuspended in PBS. Mice were randomly divided into two groups $(\mathrm{n}=6)$ for each HCC cell line. A total of $5 \times 10^{6}$ cells $/ \mathrm{mL}$ in $0.10 \mathrm{~mL}$ PBS were then injected into the liver of each nude mouse. After 4-5 weeks of monitoring, the mice were sacrificed. Tumor weight was measured, and tumor specimens were used for subsequent histological examination. After the pretreatment of the xenograft tumor specimens, the presence 
of VM was determined by CD31/PAS double staining. The expressions of MMP-14, MMP-2, VE-cadherin, E-cadherin and vimentin, which are considered to be VM markers, were detected by immunohistochemistry staining as previously described.

\section{Statistical Analysis}

All statistical analyses were performed using SPSS 19.0 for Windows (SPSS, Chicago, IL) and GraphPad Prism 5.0 (San Diego, CA, USA). The Chi-squared test was used to analyze the correlation between the expression of CD276 and clinical characteristics, and VM. Student's $t$-tests were adopted to compare continuous variables between groups. The Kaplan Meier method was performed for survival analysis and the Log rank test for the curve comparison. The results were expressed as mean $\pm \mathrm{SD}$. A $p$-value of $<$ 0.05 was considered statistically significant.

\section{Results}

\section{Higher CD276 Expression is Associated with VM and Poor Prognosis in HCC}

To detect the expression of CD276, IHC staining was performed on HCC tissue sections from TMA; The expression level was assessed according to the previouslydescribed criteria. ${ }^{26}$ PAS and CD31 double staining were applied to investigate VM in HCC tissues. Vascular-like patterns, which were formed by tumor cells, were identified as VM (Figure 1A). In the 93 HCC samples, high expression of CD276 was found in 59 of the 93 samples (63.4\%) (Figure 1B), while VM was found in 27 of the 93 HCC samples $(29.0 \%)$. The analysis of the relationship between CD276 expression and the clinicopathological characteristics showed no significant correlation between CD276 expression and age, gender, TNM stage, tumor size and histological grade. Still, the CD276 expression significantly differed between the VM positive and negative groups $(p=0.008)$ (Table 1$)$. VM was detected in 23 out of the 59 samples $(39.0 \%)$ in the CD276 high-expression group and 4 out of 34 samples (11.8\%) in the CD276 lowexpression group. The results of the Kaplan-Meier survival analysis showed that the presence of VM and high expression of CD276 were associated with poor prognosis in human hepatocellular carcinoma. In addition, these patients had a shorter survival period than those without VM or with low CD276 expression (Figure 1C). Information concerning CD276 protein (located in cytoplasmic and membranous) in the human protein atlas showed negative and positive staining in HCC (Figure 1D). In addition, data from the Kaplan-Meier Plotter database showed that high CD276 mRNA expression was associated with worse overall survival (OS) in liver cancer patients (Figure 1E).

\section{CD276 Knockdown Inhibits the Proliferation, Migration and Invasion of HCC Cells in vitro}

Western blotting was performed to analyze the expression of CD276 in various $\mathrm{HCC}$ cell lines which were able to form tube-like structures in vitro. CD276 expression was found in all of these cell lines (Figure 2A). Huh7 and Bel7404 with higher expression were selected for subsequent experiments. To investigate the roles of CD276 down-regulation in HCC progression, lentiviral vectors containing shRNA specific to CD276 were applied to knockdown the expression of CD276 in HCC cells. Western blotting revealed that the level of CD276 in Huh-7 and Bel7404 cell lines were markedly decreased by LV-shCD276 (Figure 2B). Moreover, CCK 8 assays showed that CD276 knockdown reduced the proliferation of cells (Figure $2 \mathrm{C}$ ). In addition, transwell assay (Figure 2D) and wound-healing assay (Figure 2E) showed that the down-regulation of CD276 obviously suppressed the migration and invasion of HCC cells.

\section{CD276 Promotes the VM Formation in vitro and in vivo}

To assess the effect of CD276 on VM formation, threedimensional cultures were used. The results showed that VM in the Bel7404 and Huh7 cells was significantly suppressed by CD276 down-regulation when compared with control cells $(p<0.05)$ (Figure $3 \mathrm{~A})$. VE-cadherin is considered to be a marker of VM formation. ${ }^{28}$ The results of immunofluorescence staining analysis indicated that CD276 knockdown decreased the expression of VEcadherin in Bel7404 and Huh7 cells (Figure 3B).

In the orthotopic transplantation tumor model, CD31PAS dual staining revealed the VM structures with negative CD31 staining and positive PAS staining in the tumor xenograft. Tumor tissues that had at least one VM structure were considered as having the capacity to form VM (Figure 3C). However, VM formation was not observed in the xenografts of CD276-knock-down HCC cell group (0/ 6), while there was VM formation in two xenografts of the 
A
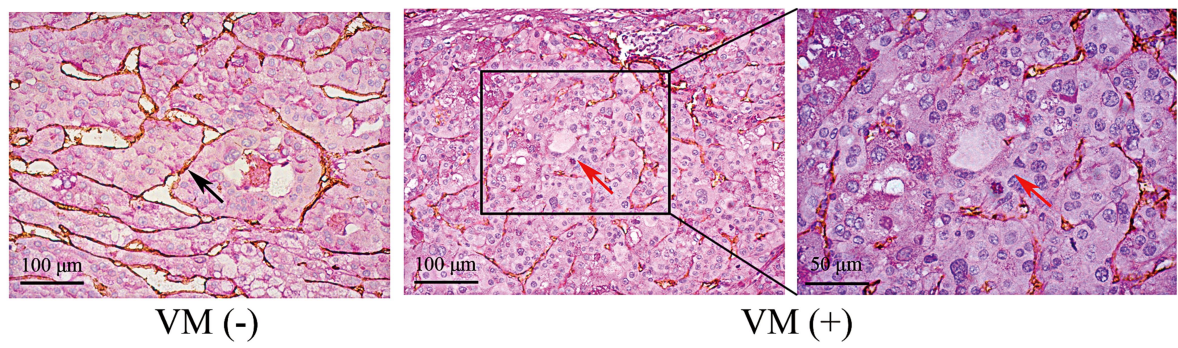

B

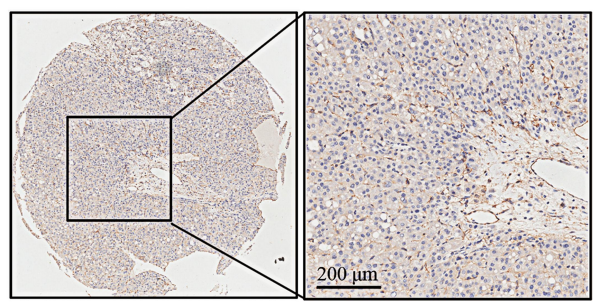

CD276 (-)

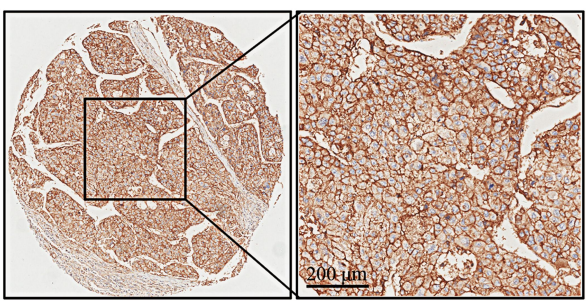

CD276 (+)

C
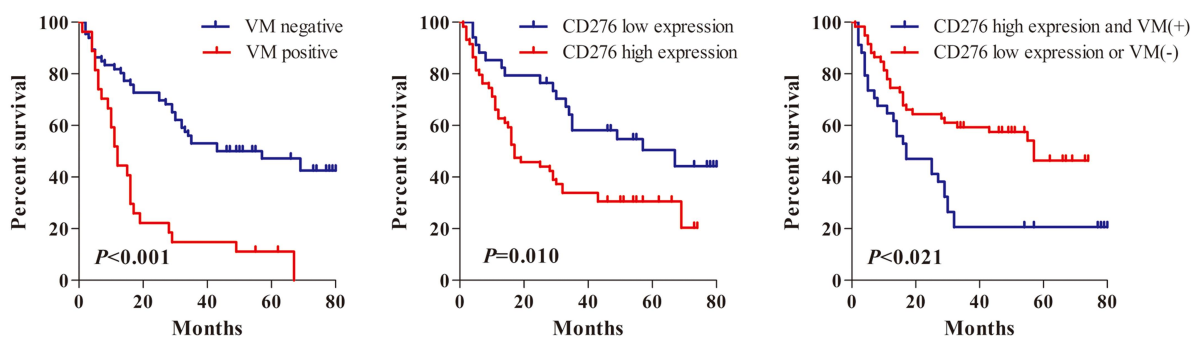

D

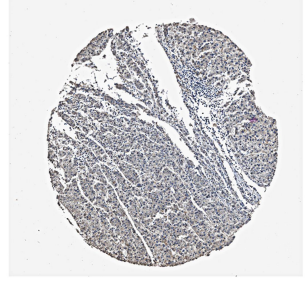

Hepatocellular carcinoma HPA017139 Staining: Not detected Intensity: Negative Quantity: None Location: None

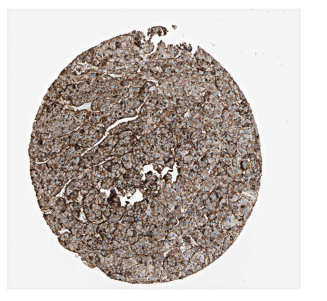

Hepatocellular carcinoma HPA009285 Staining: Medium Intensity: Moderate Quantity: $>75 \%$

Location: Cytoplasmic/membranous
E

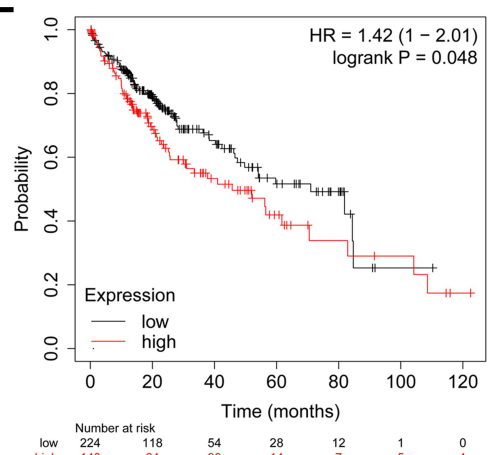

Figure I CD276 associated with vasculogenic mimicry and poor prognosis in HCC. (A) Representative images showing typical malignant morphology of vasculogenic mimicry by CD3I/PAS staining in HCC. The black arrow indicates blood vessels formed by endothelial cells (PAS-/CD3I+); The red arrows indicate the VM vessel (PAS $+/ C D 3$ I-). (B) The expression of CD276 in HCC tumor tissue microarrays was detected by immunohistochemical staining. HCC tumor sections with a final score of $\leq 3$ were defined as low CD276 expression (CD276-), whereas sections with a final score of $>3$ were defined as high CD276 expression (CD276+). (C) Kaplan-Meier survival analysis showed that patients with VM had a shorter survival period than those without $\mathrm{VM}(p<0.00 \mathrm{I})$ and those with a high CD276 expression had a shorter survival period than those with a low CD276 expression $(p=0.010)$. Patients with VM $(+)$ and high CD276 expression had worse prognosis when compared with those with VM (-) or low CD276 expression $(p=0.021)$. (D) Immunohistochemistry staining for CD276 in hepatocellular carcinoma tissue from a Human Protein Atlas Portal. (E) The prognostic value of the mRNA expression of CD276 in www.kmplot.com. Patients with liver cancer exhibiting a high mRNA expression of CD276 had a worse prognosis when compared to those with low expression levels.

negative control group (2/6, 33.3\%). Moreover, the tumor weight was significantly reduced in the mice injected with CD276-knock-down HCC cells (LV-shCD276) when compared with that in the negative control group (LV-NC) (Figure 3C).

\section{CD276 Promotes Vasculogenic Mimicry Formation via the PI3K/AKT/MMPs Pathway}

To explore the molecular mechanisms of CD276 in affecting VM formation in $\mathrm{HCC}$, we evaluated the association 
Table I Correlation Between CD276 Expression and Clinicopathologic Parameters, and VM

\begin{tabular}{|c|c|c|c|c|}
\hline \multirow[t]{2}{*}{ Factors } & \multicolumn{2}{|l|}{ CD276 } & \multirow[t]{2}{*}{$\chi^{2}$} & \multirow[t]{2}{*}{$P$} \\
\hline & $\begin{array}{l}\text { High } \\
\text { Expression }\end{array}$ & $\begin{array}{l}\text { Low } \\
\text { Expression }\end{array}$ & & \\
\hline \multicolumn{5}{|l|}{ Age (years) } \\
\hline$<60$ & 46 & 22 & 3.006 & 0.096 \\
\hline$\geq 60$ & 12 & 13 & & \\
\hline \multicolumn{5}{|l|}{ Gender } \\
\hline Male & 52 & 31 & 0.208 & $0.74 I$ \\
\hline Female & 7 & 3 & & \\
\hline \multicolumn{5}{|l|}{ TNM stage } \\
\hline $\mid / / I$ & 27 & 18 & 0.445 & 0.526 \\
\hline $\mathrm{III} / \mathrm{IV}$ & 32 & 16 & & \\
\hline \multicolumn{5}{|l|}{ Tumor size $(\mathrm{cm})$} \\
\hline$<5$ & 25 & 17 & 0.507 & 0.521 \\
\hline$\geq 5$ & 34 & 17 & & \\
\hline \multicolumn{5}{|l|}{ Histological grade } \\
\hline $\mathrm{I} / \mathrm{II}$ & 35 & 26 & 2.811 & 0.115 \\
\hline III/IV & 24 & 8 & & \\
\hline \multicolumn{5}{|l|}{ VM } \\
\hline Positive & 23 & 4 & 7.756 & $0.008^{*}$ \\
\hline Negative & 36 & 30 & & \\
\hline
\end{tabular}

Note: *Significantly different.

between CD276 and key molecules in the PI3K/AKT/ MMPs signaling pathway using the GEPIA database. These molecules included AKT1, MMP14, and MMP2. The results revealed significant correlations between CD276 expression and AKT1 $(\mathrm{R}=0.41, p=4.4 \mathrm{e}-16)$, MMP14 $(\mathrm{R}=0.7, p=0), \operatorname{MMP} 2(\mathrm{R}=0.59, p=0)$ (Figure 4A).

We further explored the effect of the down-regulated CD276 expression on the PI3K/AKT/MMPs signaling pathway in HCC cells. Constitutive activation of this signaling pathway was found in both $\mathrm{HCC}$ cell lines. Analyses of Western blotting showed that CD276 knockdown significantly inhibited AKT phosphorylation and down-regulated the expression of MMP14, MMP2, VEcadherin and vimentin. In contrast, the expression of E-cadherin was up-regulated (Figure 4B). Moreover, the gelatin zymography assay showed that MMP2 and MMP9 activities were down-regulated in the RNA interference group, when compared with the negative control (Figure 4C). LY294002 significantly inhibited the phosphorylation of AKT, while the expression of CD276 was not significantly changed, which suggested that CD276 functions as the upstream of AKT. We also found that the treatment with LY294002 alone or in combination with LV-shCD276 effectively blocked the cell invasion, migration, and tube formation. However, the inhibitive effect of LY294002 alone on the tube formation was less favorable than using LV-shCD276 alone or in combination with LY294002 (Figure 5A-C).

In addition, IHC analysis revealed a decreased expression of MMP-14, MMP2 VE-cadherin, vimentin and increased expression of E-cadherin in the xenograft tumor tissue of the CD276-knock-down HCC cell group when compared with those of the control cell group (Figure 5D). These results indicated that CD276 expression may influence VM formation in vitro and in vivo through the PI3K/AKT/MMPs signaling pathway.

\section{Discussion}

Vasculogenic mimicry is associated with malignancy and poor clinical prognosis in $\mathrm{HCC}^{8}$ Given the association between aggressive tumors and $\mathrm{VM}$, it is necessary to seek VM-targeted therapies in the light of their formation and induction mechanism.

Previous studies have shown that CD276 is aberrantly expressed in HCC cells. Its high expression can promote the proliferation, adhesion, migration, and invasion capacity of tumor cells. ${ }^{18,29}$ Furthermore, CD276 is identified as a cell-surface tumor endothelial marker that can distinguish pathological and physiological angiogenesis. ${ }^{30}$ A recent study has shown that antibody-drug conjugates (ADCs) with a conventional pyrrolobenzodiazepine warhead targeting CD276 may eradicate both cancer cells and tumor vasculature, destroying large established tumors and metastases, and improving the long-term overall survival. $^{31}$ Therefore, CD276 seems to be an ideal target for anti-vascular therapy that simultaneously destroys both tumor endothelial vessels and VM. Yet, the relationship between CD276 and VM has not been reported so far.

In this study, we found that CD276 protein expression was associated with the presence of VM and poor prognosis of HCC patients. Consequently, we transfected a CD276-targeting shRNA lentiviral vector into two HCC cell lines (Huh7 and Bel7404) to further examine its effect on cell biological behavior in vitro and in vivo. The Western blotting analysis showed that CD276 protein expression was significantly down-regulated in both cell lines. Moreover, CD276 knockdown decreased the proliferation, invasion, migration, and tube formation in vitro. VE-cadherin is usually present during VM formation in 
A

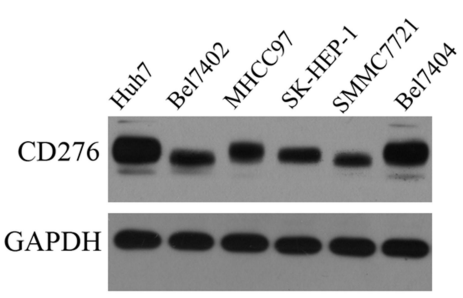

B

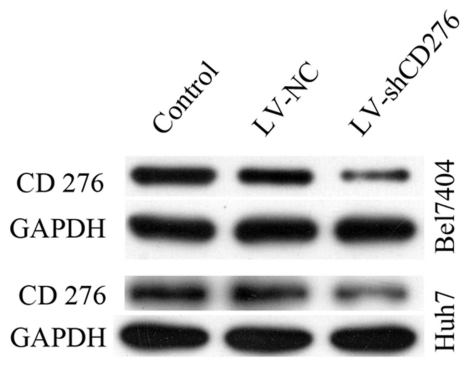

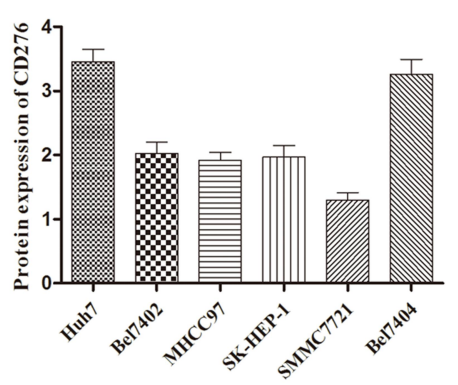

C

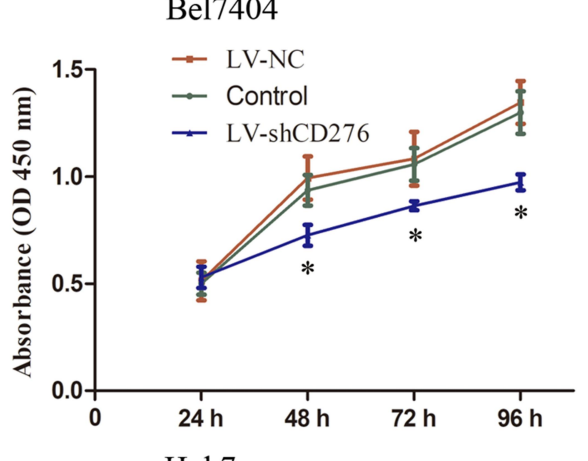

Huh7
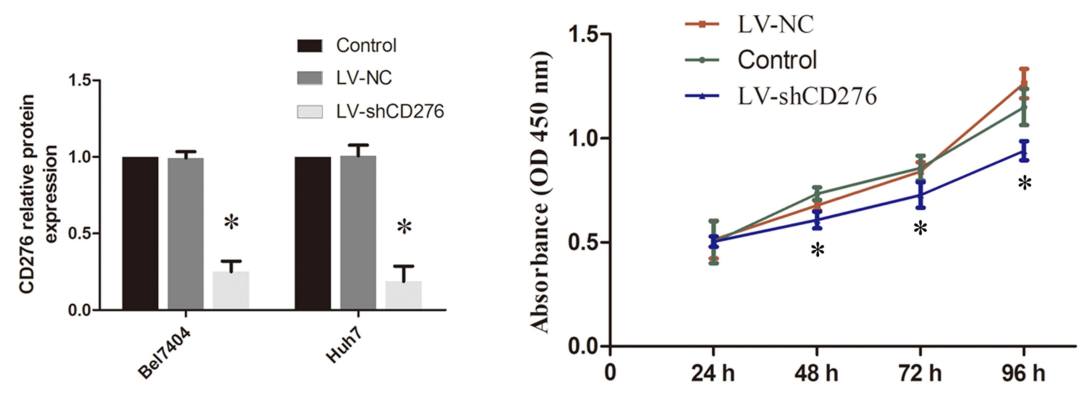

D

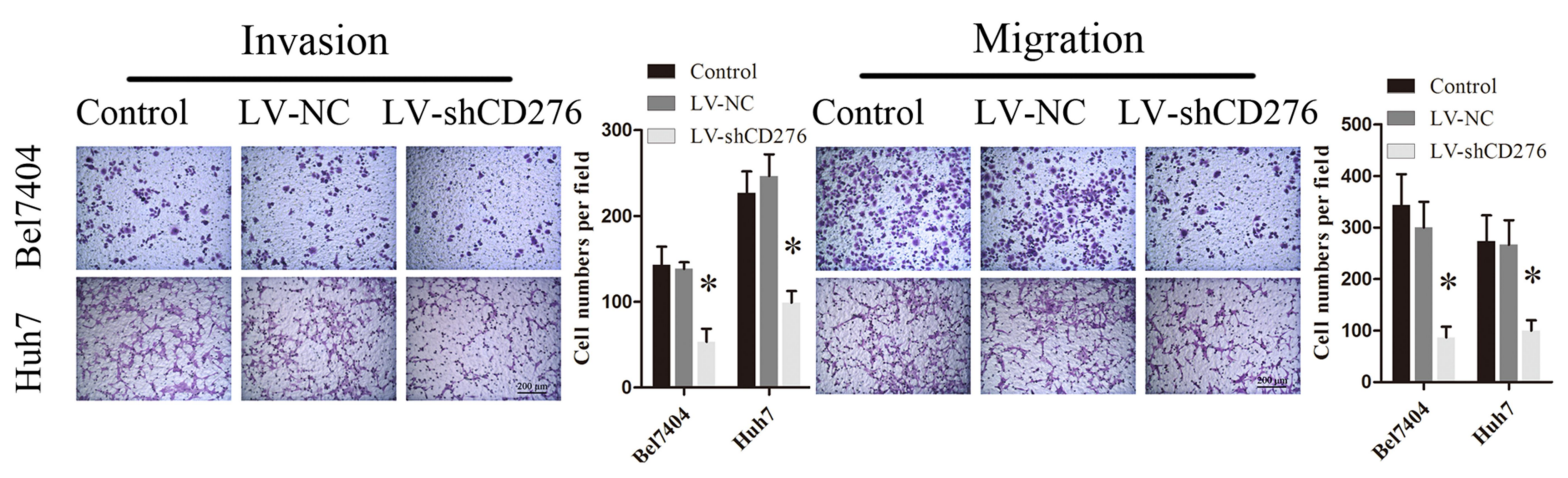

$\mathbf{E}$
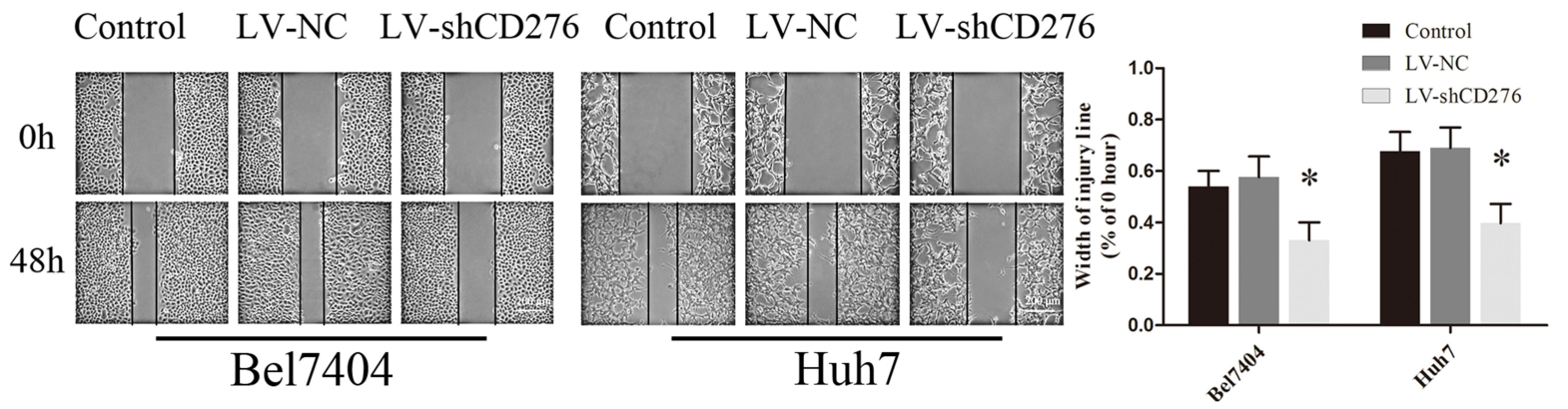

Figure 2 CD276 knock-down led to less HCC cells proliferation, invasion and migration in vitro. (A) Endogenous expression of CD276 in various human HCC cell lines by Western blotting. CD276 was highly expressed in Bel7404 and Huh7 cells and the two cell lines were selected for subsequent experiments. (B) Bel7404 and Huh7 cells were transfected with lentiviral vectors containing shRNA specific to CD276 (LV-shCD276) and a non-targeted control shRNA (LV-NC). Cells without any treatment were used as blank control (Control). The protein expression of CD276 in cells was evaluated by Western blotting. (C) CCK8 proliferation assay demonstrated that CD276 knockdown inhibited the cellular proliferation ability in HCC cell lines $\left({ }^{*} p<0.05\right)$. (D) The invasion and migration assays of down-regulated CD276 expression in Bel7404 and Huh7 cells were performed with Transwell chambers. The representative images and the quantification of the results are presented as mean \pm SD; Scale bar represents $200 \mu \mathrm{m}\left({ }^{*} p<0.05\right)$. (E) Wound-healing assays of down-regulated CD276 expression in Bel7404 and Huh7 cells were performed. The representative images and the quantification are presented as mean $\pm \mathrm{SD}$; Scale bar represents $200 \mu \mathrm{m}\left({ }^{*} p<0.05\right)$.

tumor cells and is a significant marker for VM, exclusively expressed in highly aggressive tumor cells. ${ }^{10}$ Furthermore, we established an orthotopic xenograft model of HCC cells and observed less VM formation in mice injected with CD276-knock-down HCC cells. These data suggested that CD276 expression might have an essential role in VM 
A

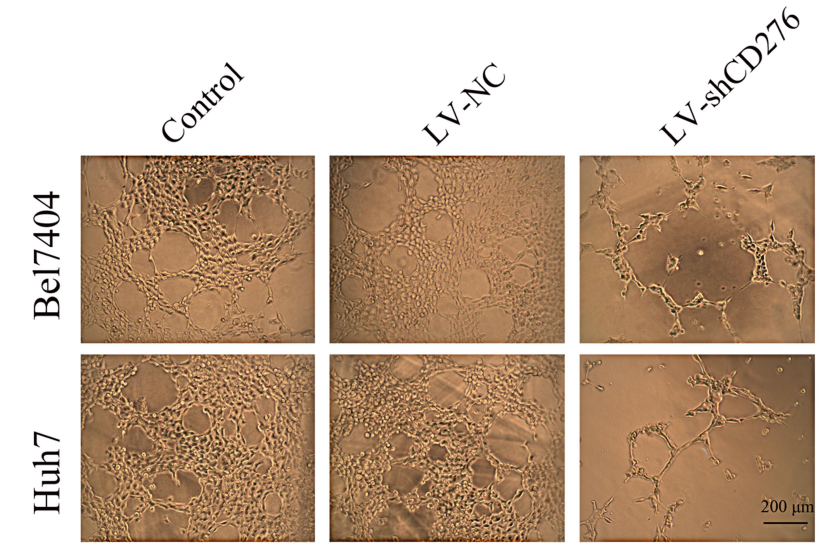

B

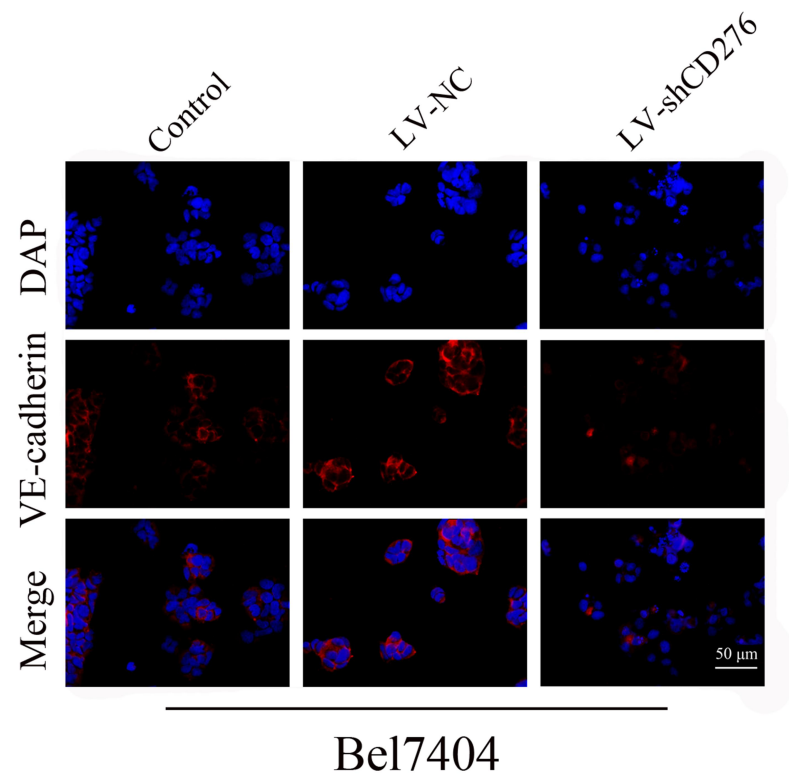

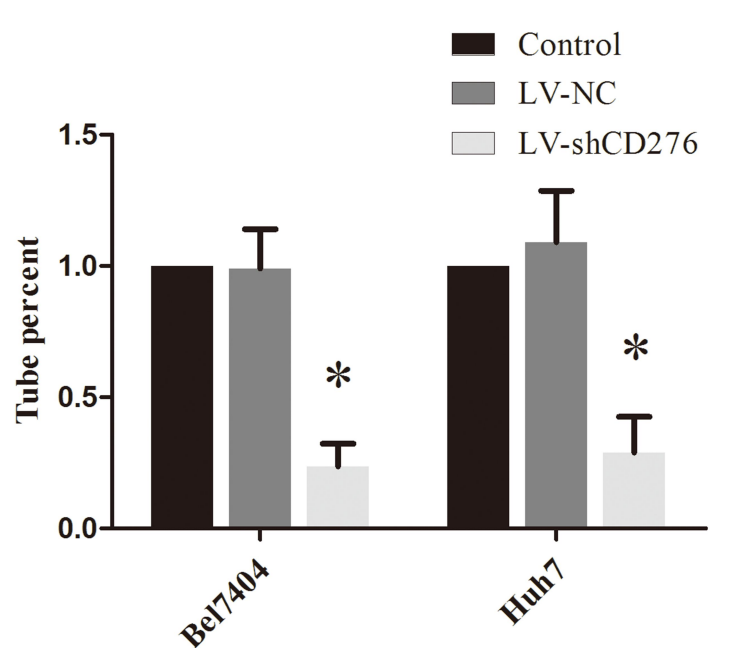

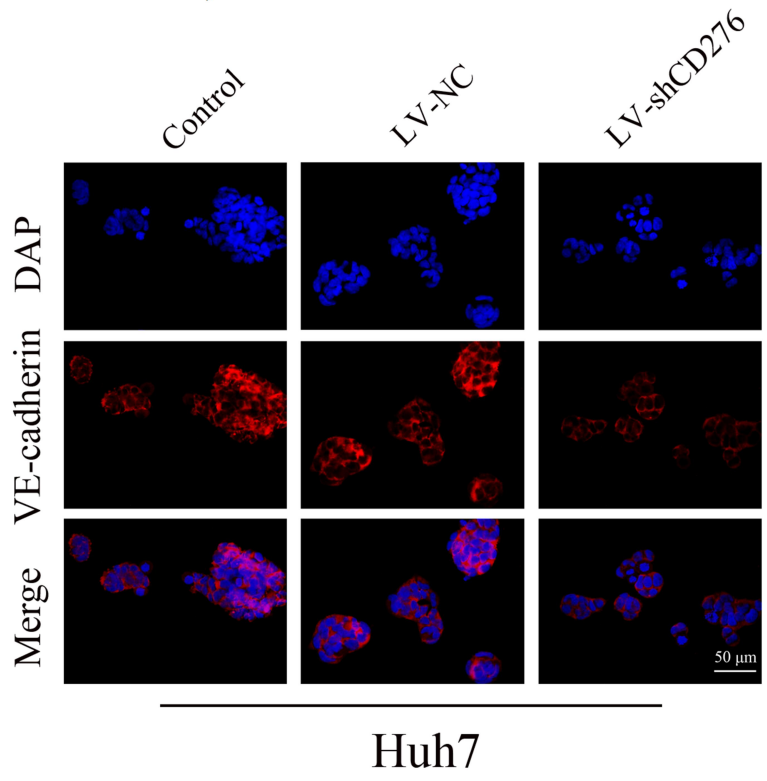

$50 \mu \mathrm{m}$
C

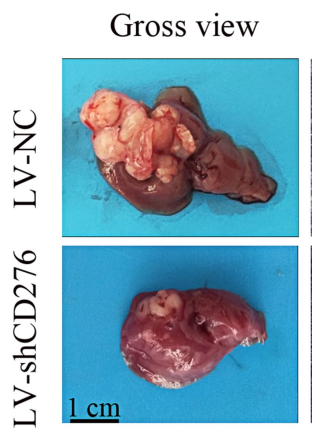

HE staining

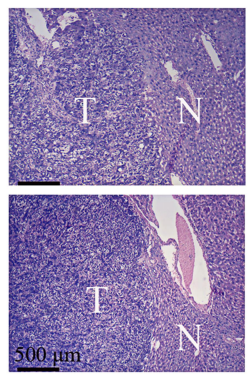

PAS/CD31 double staining

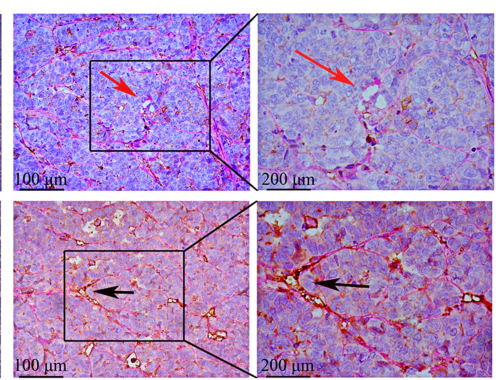

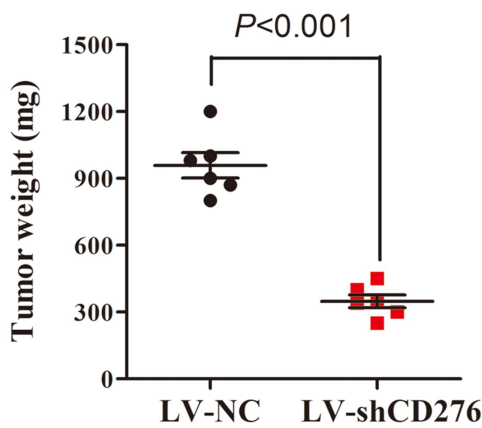

Figure 3 RNA interference of CD276 inhibited VM formation of HCC cell lines in vitro and in vivo. (A) The 3D VM formation assay was performed to analyze the formation of the vascular-like tube in HCC cells. The representative images and the quantification of the results are presented as mean \pm SD; scale bar, 200 $\mu \mathrm{m}\left({ }^{*} p<0.05\right)$; (B) Immunofluorescence analyses showed that CD276 knock-down decreased the expression of VE-cadherin in Bel7404 and Huh-7cells; Scale bar represents $50 \mu \mathrm{m}$. Original magnification: 400x; (C) CD276 knock-down inhibited VM formation in an orthotopic transplant mouse model of HCC cells. The letter "T" represents tumor tissue and " $\mathrm{N}$ " represents normal liver tissue. The black arrows indicate blood vessels formed by endothelial cells (PAS-/CD3I+) and the red arrows indicate the VM vessel (PAS+/CD3I-). In addition, CD276 knock-down also led to a reduced tumor weight. The representative images and the quantification of the results are presented as mean \pm SD $(* p<0.01)$. 
A
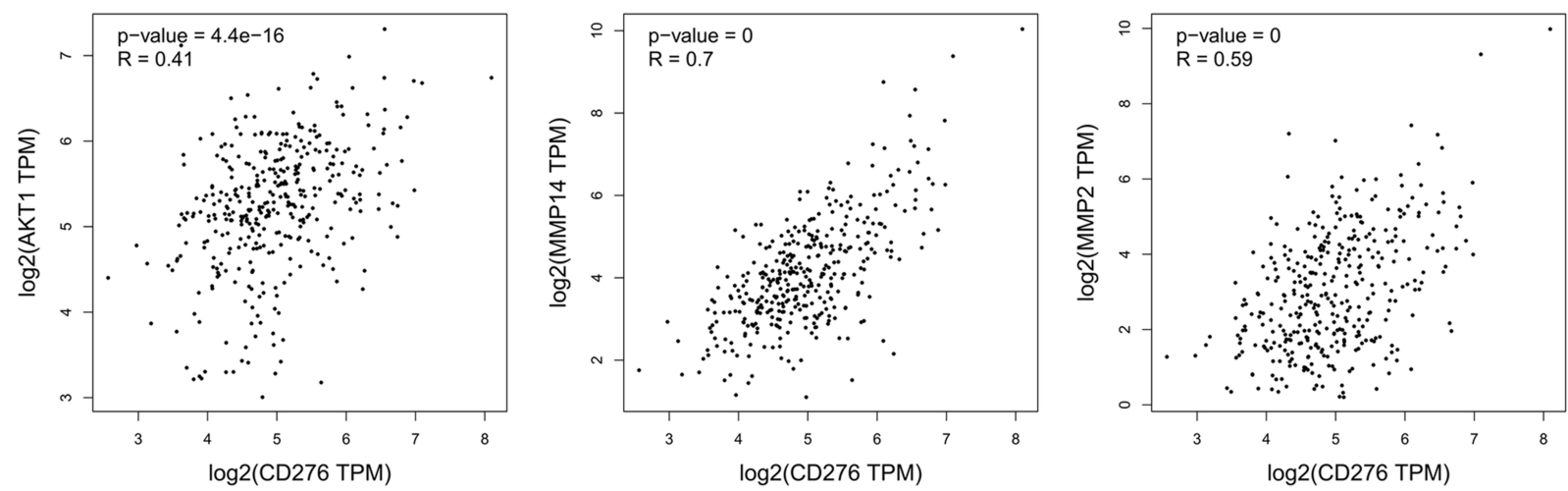

B
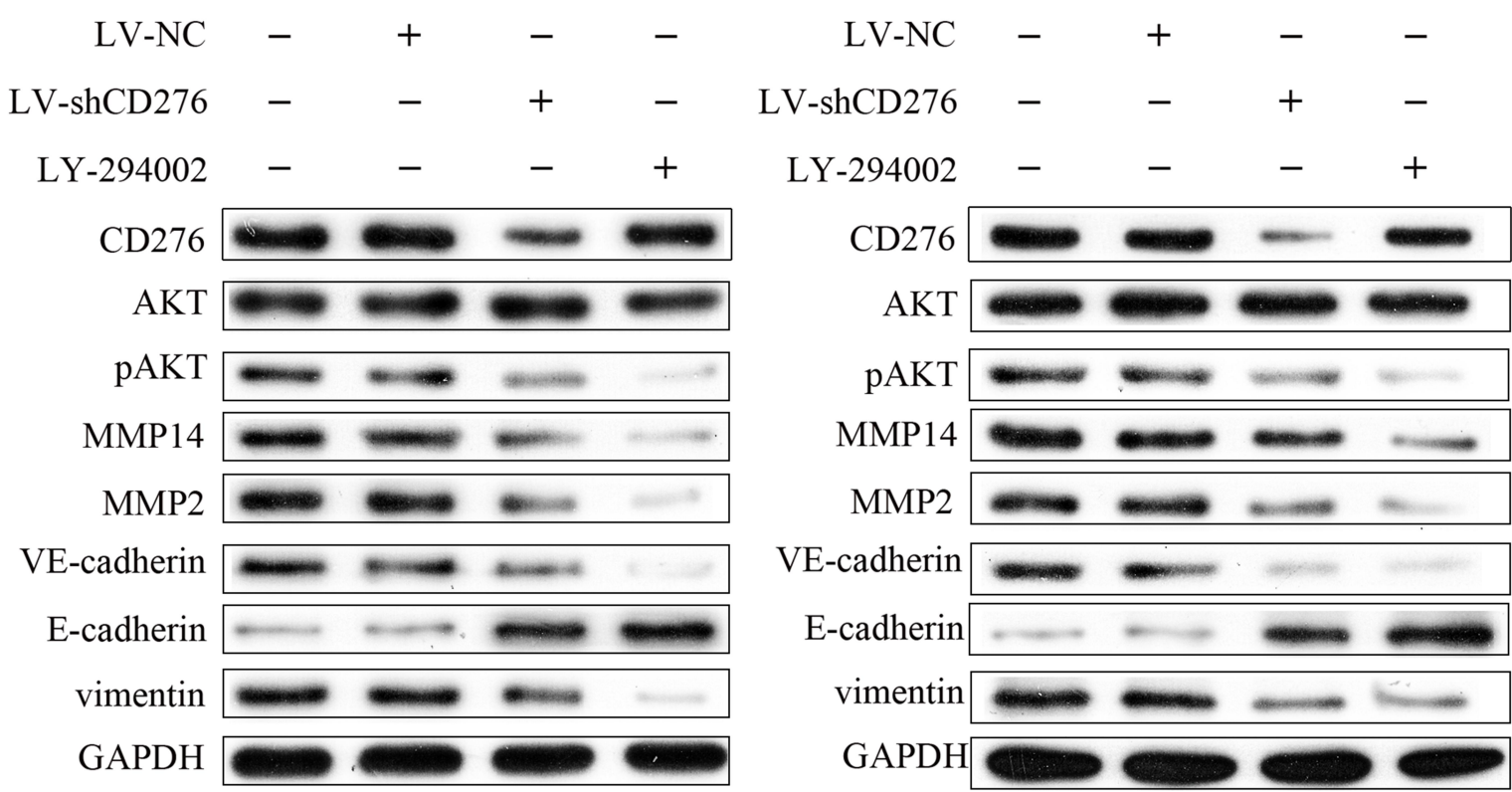

Bel7404

\section{Huh7}

C
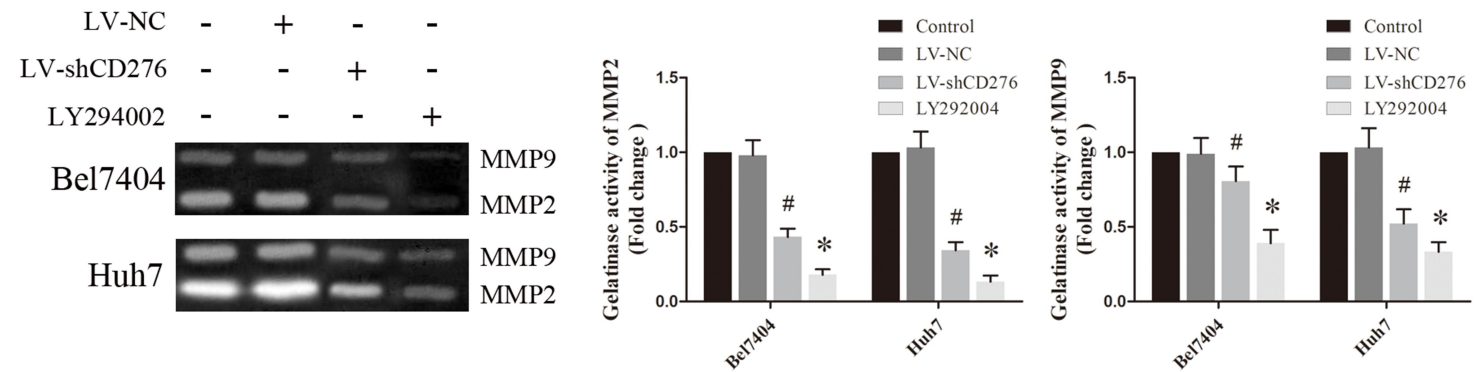

Figure 4 CD276 promoted vasculogenic mimicry formation via the PI3K/AKT/MMPs pathway and EMT induction in HCC. (A) Correlations between CD276 and key molecules of the PI3K/AKT/MMPs pathway were analyzed using the GEPIA database. (B) Bel7404 (left) and Huh7 (right) cells were pretreated with LV-NC or LV-shCD276 or LY294002 $(20 \mu \mathrm{M})$ and protein levels of CD276, AKT, p-AKT, MMPI4, MMP2, VE-cadherin, E-cadherin and vimentin were then detected by Western blotting. (C) Activation of MMP2 and MMP9 was evaluated by gelatin zymography and their activities were both down-regulated in the RNA interference group, as compared with the negative control $\left({ }^{*} p<0.05 ;{ }^{*} p<0.05\right)$.

formation in HCC. However, the underlying mechanisms though which CD276 expression affects the formation of VM remain unclear.
Previous studies have suggested that CD276 may promote tumor aggression and invasion by regulating the expression and activities of matrix metalloproteinases 
A

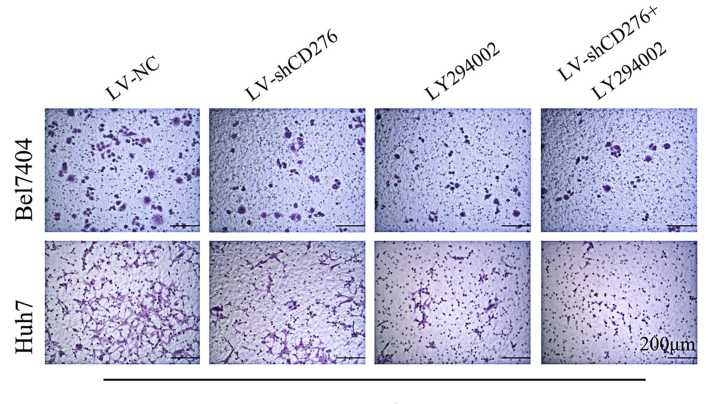

Invasion

B

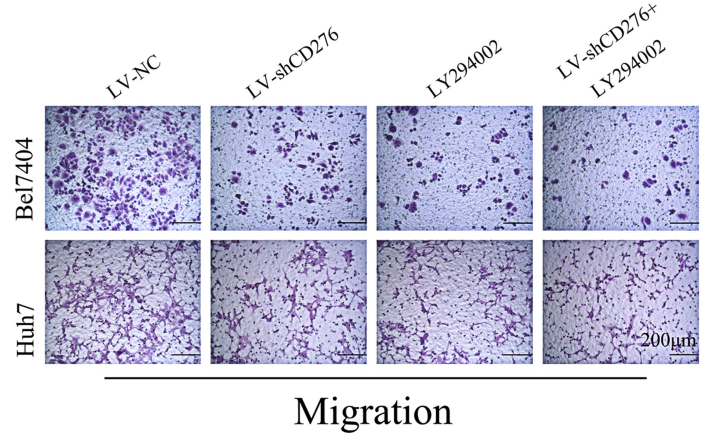

C
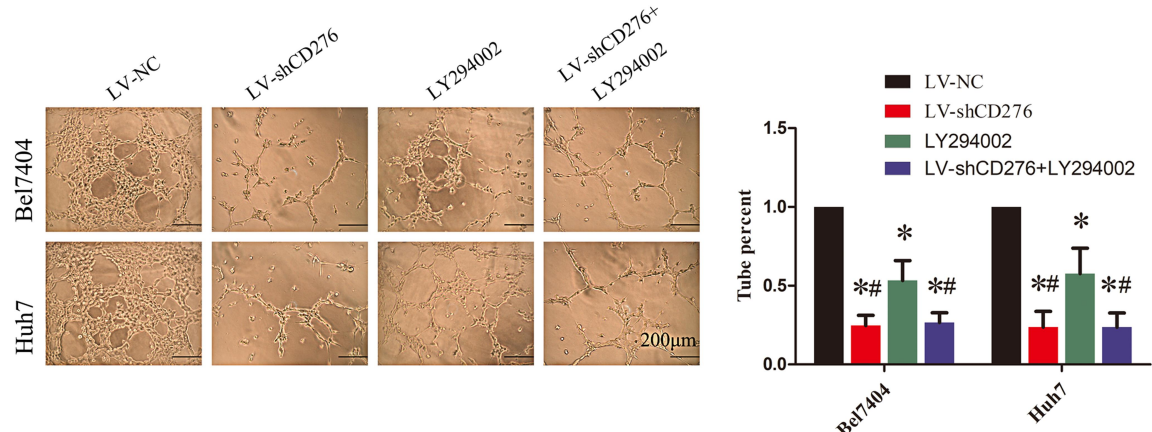

D
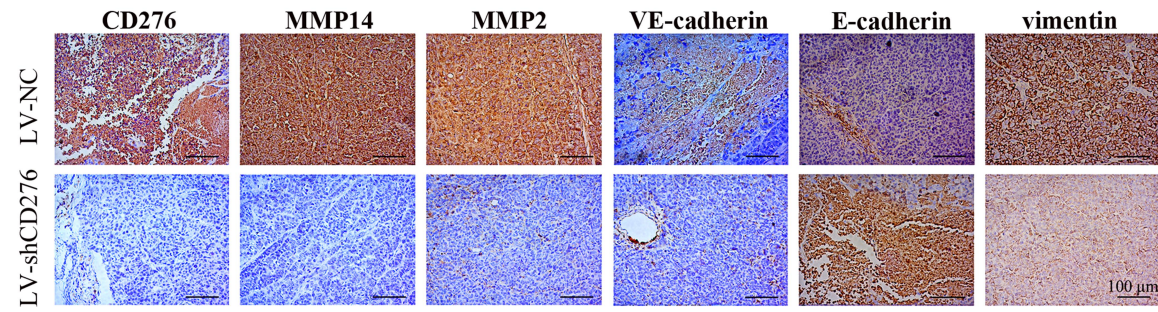

Figure 5 The activation of the PI3K/AKT/MMPs signaling pathway and EMT might be important mechanisms involved in CD276-promoting VM formation in HCC. (A) LY294002, a PI3K inhibitor, suppressed the invasion of HCC cells in vitro, just as CD276 knockdown did. The representative images and the quantification of the results are presented as mean \pm SD; Scale bar represents $200 \mu \mathrm{m}\left({ }^{*} p<0.05\right)$. (B) Tanswell migration assay showed that LY294002 also suppressed the migration of HCC cells in vitro. The representative images and the quantification of the results are presented as mean \pm SD; Scale bar represents $200 \mu \mathrm{m}\left({ }^{*} p<0.05\right)$. (C) LY294002 inhibited the tube formation of Bel7404 and Huh-7 cells in vitro, as CD276 knockdown did. However, the inhibitive effect of LY294002 alone on the tube formation is less favorable than using LV-shCD276 alone or in combination with LY294002. The representative images and the quantification of the results are presented as mean \pm SD; Scale bar represents $200 \mu m\left({ }^{*} p<0.05\right.$, compare to LV-NC treatment group, respectively; ${ }^{\#} p<$ 0.05, compare to LY292004 treatment group, respectively). (D) Immunohistochemical analysis showed that CD276 knock-down decreased the protein levels of MMPI4, MMP2, VEcadherin, vimentin, and increased the protein expression of E-cadherin in the orthotopic transplant mouse model of HCC cells.

(MMPs). ${ }^{32,33}$ MMPs are a broad family of zinc-biding endopeptidases that are implicated in a variety of mechanisms promoting tumor progression. ${ }^{34}$ Specifically, MMP2 and MMP14 are reported to be significantly related to VM formation in gallbladder carcinoma. ${ }^{35}$ MMP2 exerts a strong proteolytic effect on the extracellular matrix.
MMP14, also known as membrane type-1 matrix metalloproteinase (MT1-MMP), can enhance collagen IV degradation by forming a complex with the tissue inhibitor of metalloproteinase 2 and activating pro-MMP2. ${ }^{36}$ In the initial step of VM formation, the invading cancer cells may lose cell-cell adhesion, increase their motility, and 
secrete MMPs to dissolve the basal membrane. ${ }^{37}$ It has been reported that MMP2 or MMP14 antibodies can inhibit VM formation. ${ }^{35}$ In this study, the expression and activation of MMPs in HCC cells were examined by Western blotting and gelatin zymography assay. The results showed that CD276 protein expression was positively correlated with MMP14/MMP2 expression and MMP2/MMP9 activation, thus suggesting that CD276 may affect the VM formation by regulating the expression and activity of MMPs in HCC cells.

As the PI3K/AKT/MMPs signaling pathways have been reported as a key regulatory pathway of VM formation in gallbladder carcinomas and melanoma, ${ }^{35,38}$ we suspect that CD276 may also affect VM formation through the PI3K/AKT/MMPs signaling pathway. To examine this hypothesis, we explored the correlation between CD276 and key molecules of this signaling pathway in GEPIA and found a significant positive correlation. Then, we down-regulated the expression of CD276 expression in HCC cells in vitro by using lentivirus-mediated RNA interference. As a result, the inhibition of AKT phosphorylation and down-regulation of MMP2 and MMP14 expression were evident. Subsequently, we used the PI3K inhibitor LY294002 to assess its effect on the VM formation of HCC. The results showed that LY294002 significantly inhibited the phosphorylation of AKT and blocked the effect of cell invasion, migration, tube formation, but did not significantly change the expression level of CD276. Therefore, we propose that CD276 may function as the upstream of AKT. Interestingly, we found that CD276 knock-down seems to have a better inhibitory effect on tube formation than LY294002 alone. A reasonable explanation for this may be that downregulating CD276 to inhibit the formation of vasculogenic mimicry may also involve other molecular pathways, which should be further addressed by subsequent studies.

The expression of VE-cadherin and vimentin was down-regulated in the cells with CD276 knockdown, whereas the expression of E-cadherin was up-regulated. These findings indicate that the effect CD276 on VM formation in HCC may also involve epithelial-tomesenchymal transition (EMT). EMT describes a special process in which epithelial cells lose many of their epithelial characteristics and take on properties typical of mesenchymal cells. It has a vital role in the malignant progression and metastasis of tumors. ${ }^{39}$ Previous studies have demonstrated that changes in related phenotypic genes are often associated with the development of EMT in epithelial tumor cells, such as a decrease in the epithelial marker E-cadherin and an increase in the mesenchymal marker vimentin. ${ }^{39}$ EMT has an important role in the formation of $\mathrm{VM}^{12}$ Furthermore, a previous study reported that $\mathrm{CD} 276$ promotes aggression and invasion of HCC by targeting EMT. ${ }^{26}$ Moreover, MMPs are key mediators for the EMT process and tumor progression. ${ }^{40}$ It has been suggested that the implication of MMPs in EMT and tumor progression may involve three mechanisms: (a) increased MMP levels in the tumor microenvironment can directly promote EMT in epithelial cells; (b) tumor cells undergoing EMT can synthesize more MMPs in order to initiate the invasion and metastasis processes; (c) EMT can affect peritumoral cells that participate in tumor progression to further produce MMPs. ${ }^{41}$ In light of the above data, it is plausible that $\mathrm{CD} 276$ promotes VM formation by activating the PI3K/AKT/MMPs signaling pathway and the induction of EMT.

In summary, our study shows that CD276 promotes vasculogenic mimicry formation via PI3K/AKT/MMPs pathway in hepatocellular carcinoma. Down-regulation of CD276 can inhibit the activation of the PI3K/AKT/MMPs pathway and down-regulate the expression and activity of MMPs in HCC cells, leading to the suppression of tumor aggressiveness and the EMT process, which are key regulators of VM formation. These findings provide important clues into the regulatory mechanisms underlying VM and specifically identify CD276 as a potential new target for the anti-vascular treatment of hepatocellular carcinoma.

\section{Abbreviations}

VM, vasculogenic mimicry; HCC, hepatocellular carcinoma; MMPs, matrix metalloproteinases; PAS, periodic acid-Schiff; TMA, tissue microarray; VE-cadherin, vascular endothelial cadherin; EMT, epithelial-to-mesenchymal transition; EDTA, ethylenediaminetetraacetic acid; DAB, 3,3'-diaminobenzidine chromogen; EGFP, enhanced green fluorescent protein; shRNA, short hairpin RNA; MOI, multiplicity of infection; FBS, fetal bovine serum; PBS, phosphate buffer saline; MR, migration rate; GEPIA, Gene Expression Profiling Interactive Analysis; TCGA, The Cancer Genome Atlas; RIPA, radio-immunoprecipitation assay; BCA, bicinchoninic acid; pAKT, phosphorylated AKT; PVDF, polyvinylidene difluoride; GAPDH, glyceraldehyde-3-phosphate dehydrogenase; ADCs, antibody drug conjugates; MT1-MMP, membrane type-1 matrix metalloproteinase. 


\section{Ethics Approval and Consent to Participate}

This study was permitted and supervised by the ethics committee of Fujian Medical University Union Hospital. The use of the tissue microarrays was approved by the Ethics Committee of Shanghai Outdo Biotech (Ethical approval number: T19-0361); The protocol was accepted by Animal Welfare Committee of Fujian Medical University. All experiments involving animals were carried out following the Guide for the Care and Use of Laboratory animals published by the National Institutes of Health. Noteworthy efforts were made to curtail the number of animals and their sufferings.

\section{Author Contributions}

All authors made substantial contributions to conception and design, acquisition of data, or analysis and interpretation of data; took part in drafting the article or revising it critically for important intellectual content; agreed on the journal to which the article will be submitted; gave final approval of the version to be published; and agree to be accountable for all aspects of the work. All the authors have made significant contributions and agree with the content of the manuscript.

\section{Funding}

This study was supported by Joint Funds for Innovative Science and Technology, Fujian Province [Grant number: 2017Y9022]; Startup Fund for Scientific Research, Fujian Medical University [Grant number: 2017XQ1046]; Funds for Young and Middle-Aged Talents of Fujian Health Care System sponsored by Fujian provincial health technology project [Grant number: 2019-ZQN-40 and 2016-ZQN-35]; Natural Science Foundation of Fujian Province [Grant number: 2017J01206]; Middle-aged Young Teachers' Education Scientific Research Projects in Fujian Province, China [Grant number: JAT170221].

\section{Disclosure}

The authors report no conflicts of interest for this work.

\section{References}

1. Gao J, Wang Z, Wang GJ, et al. Higher CYP2E1 activity correlates with hepatocarcinogenesis induced by diethylnitrosamine. J Pharmacol Exp Ther. 2018;365(2):398-407. doi:10.1124/jpet.117.245555

2. Kudo M. Systemic therapy for hepatocellular carcinoma: 2017 update. Oncology. 2017;93(Suppl 1):135-146. doi:10.1159/000481244

3. Siegel RL, Miller KD, Jemal A. Cancer statistics, 2019. CA Cancer J Clin. 2019;69(1):7-34.
4. Hao CY. Angiogenesis blockade as therapy for hepatocellular carcinoma: progress and challenges. J Gastroenterol Hepatol. 2011;26 (1):4-6.

5. Plate KH, Scholz A, Dumont DJ. Tumor angiogenesis and anti-angiogenic therapy in malignant gliomas revisited. Acta Neuropathol. 2012;124(6):763-775.

6. Folkman J. Proceedings: tumor angiogenesis factor. Cancer Res. 1974;34(8):2109-2113.

7. Shen Y, Quan J, Wang M, et al. Tumor vasculogenic mimicry formation as an unfavorable prognostic indicator in patients with breast cancer. Oncotarget. 2017.

8. Sun B, Zhang S, Zhang D, et al. Vasculogenic mimicry is associated with high tumor grade, invasion and metastasis, and short survival in patients with hepatocellular carcinoma. Oncol Rep. 2006;16 (4):693-698.

9. Sun T, Zhao N, Zhao XL, et al. Expression and functional significance of Twist1 in hepatocellular carcinoma: its role in vasculogenic mimicry. Hepatology. 2010;51(2):545-556. doi:10.1002/hep.23311

10. Cao Z, Sun B, Zhao X, et al. The expression and functional significance of runx 2 in hepatocellular carcinoma: its role in vasculogenic mimicry and epithelial-mesenchymal transition. Int $\mathrm{J} \mathrm{Mol} \mathrm{Sci.}$ 2017;18(3):500. doi:10.3390/ijms18030500

11. Folberg R, Maniotis AJ. Vasculogenic mimicry. APMIS. 2004;112 (7-8):508-525. doi:10.1111/j.1600-0463.2004.apm11207-0810.x

12. Sun B, Zhang D, Zhao N, Zhao X. Epithelial-to-endothelial transition and cancer stem cells: two cornerstones of vasculogenic mimicry in malignant tumors. Oncotarget. 2017;8(18):30502-30510. doi:10.18 632/oncotarget.8461

13. Greenwald RJ, Freeman GJ, Sharpe AH. The B7 family revisited. Annu Rev Immunol. 2005;23(1):515-548. doi:10.1146/annurev. immunol.23.021704.115611

14. Picarda E, Ohaegbulam KC, Zang X. Molecular pathways: targeting B7-H3 (CD276) for human cancer immunotherapy. Clin Cancer Res. 2016;22(14):3425-3431. doi:10.1158/1078-0432.CCR-15-2428

15. Altan M, Pelekanou V, Schalper KA, et al. B7-H3 expression in NSCLC and its association with B7-H4, PD-L1 and tumor-infiltrating lymphocytes. Clin Cancer Res. 2017;23 (17):5202-5209. doi:10.1158/1078-0432.CCR-16-3107

16. Mao Y, Chen L, Wang F, et al. Cancer cell-expressed B7-H3 regulates the differentiation of tumor-associated macrophages in human colorectal carcinoma. Oncol Lett. 2017;14(5):6177-6183.

17. Xie C, Liu D, Chen Q, Yang C, Wang B, Wu H. Soluble B7-H3 promotes the invasion and metastasis of pancreatic carcinoma cells through the TLR4/NF-kappaB pathway. Sci Rep. 2016;6(1):27528. doi: $10.1038 /$ srep 27528

18. Sun TW, Gao Q, Qiu SJ, et al. B7-H3 is expressed in human hepatocellular carcinoma and is associated with tumor aggressiveness and postoperative recurrence. Cancer Immunol Immunother. 2012;61 (11):2171-2182. doi:10.1007/s00262-012-1278-5

19. Dong P, Xiong Y, Yue J, Hanley SJB, Watari H. B7H3 as a promoter of metastasis and promising therapeutic target. Front Oncol. 2018;8:264.

20. Flem-Karlsen K, Tekle C, Andersson Y, Flatmark K, Fodstad O, Nunes-Xavier CE. Immunoregulatory protein B7-H3 promotes growth and decreases sensitivity to therapy in metastatic melanoma cells. Pigment Cell Melanoma Res. 2017;30(5):467-476. doi:10.1111/pcmr.12599

21. Li Y, Yang X, Wu Y, et al. B7-H3 promotes gastric cancer cell migration and invasion. Oncotarget. 2017;8(42):71725-71735. doi:10.18632/oncotarget. 17847

22. Feng $\mathrm{P}$, Zhang H, Zhang Z, et al. The interaction of MMP-2/B7-H3 in human osteoporosis. Clin Immunol. 2016;162:118-124. doi:10.1016/ j.clim.2015.11.009

23. Tekle C, Nygren MK, Chen YW, et al. B7-H3 contributes to the metastatic capacity of melanoma cells by modulation of known metastasis-associated genes. Int J Cancer. 2012;130(10):2282-2290. doi:10.1002/ijc. 26238 
24. Meng J, Chen S, Lei YY, et al. Hsp90beta promotes aggressive vasculogenic mimicry via epithelial-mesenchymal transition in hepatocellular carcinoma. Oncogene. 2019;38(2):228-243. doi:10.1038/ s41388-018-0428-4

25. Cheng R, Cai XR, Ke K, Chen YL. Notch4 inhibition suppresses invasion and vasculogenic mimicry formation of hepatocellular carcinoma cells. J Huazhong Univ Sci Tech Med Sci. 2017;37 (5):719-725.

26. Kang FB, Wang L, Jia HC, et al. B7-H3 promotes aggression and invasion of hepatocellular carcinoma by targeting epithelial-tomesenchymal transition via JAK2/STAT3/Slug signaling pathway. Cancer Cell Int. 2015;15(1):45. doi:10.1186/s12935-015-0195-Z

27. Tang Z, Li C, Kang B, Gao G, Li C, Zhang Z. GEPIA: a web server for cancer and normal gene expression profiling and interactive analyses. Nucleic Acids Res. 2017;45(W1):W98-W102. doi:10.10 93/nar/gkx247

28. Zhao X, Sun B, Liu T, et al. Long noncoding RNA n339260 promotes vasculogenic mimicry and cancer stem cell development in hepatocellular carcinoma. Cancer Sci. 2018;109(10):3197-3208. doi: $10.1111 /$ cas. 13740

29. Wang F, Wang G, Liu T, Yu G, Zhang G, Luan X. B7-H3 was highly expressed in human primary hepatocellular carcinoma and promoted tumor progression. Cancer Invest. 2014;32(6):262-271. doi:10.3109/ 07357907.2014 .909826

30. Seaman S, Stevens J, Yang MY, Logsdon D, Graff-Cherry C, St Croix B. Genes that distinguish physiological and pathological angiogenesis. Cancer Cell. 2007;11(6):539-554. doi:10.1016/j.ccr.20 07.04.017

31. Seaman S, Zhu Z, Saha S, et al. Eradication of tumors through simultaneous ablation of CD276/B7-H3-positive tumor cells and tumor vasculature. Cancer Cell. 2017;31(4):501-515e508. doi:10.10 16/j.ccell.2017.03.005

32. Jiang B, Zhang T, Liu F, et al. The co-stimulatory molecule B7-H3 promotes the epithelial-mesenchymal transition in colorectal cancer. Oncotarget. 2016;7(22):31755-31771.
33. Xu L, Ding X, Tan H, Qian J. Correlation between B7-H3 expression and matrix metalloproteinases 2 expression in pancreatic cancer. Cancer Cell Int. 2013;13(1):81. doi:10.1186/1475-2867-13-81

34. Li K, Tay FR, Yiu CKY. The past, present and future perspectives of matrix metalloproteinase inhibitors. Pharmacol Ther. 2019;107465.

35. Lu XS, Sun W, Ge CY, Zhang WZ, Fan YZ. Contribution of the PI3K/MMPs/Ln-5gamma2 and EphA2/FAK/Paxillin signaling pathways to tumor growth and vasculogenic mimicry of gallbladder carcinomas. Int J Oncol. 2013;42(6):2103-2115. doi:10.3892/ijo.20 13.1897

36. Zhang JG, Zhang DD, Liu Y, et al. RhoC/ROCK2 promotes vasculogenic mimicry formation primarily through ERK/MMPs in hepatocellular carcinoma. Biochim Biophys Acta Mol Basis Dis. 2019;1865 (6):1113-1125. doi:10.1016/j.bbadis.2018.12.007

37. Zhu W, Sun W, Zhang JT, Liu ZY, Li XP, Fan YZ. Norcantharidin enhances TIMP2 antivasculogenic mimicry activity for human gallbladder cancers through downregulating MMP2 and MT1MMP. Int J Oncol. 2015;46(2):627-640. doi:10.3892/ijo.2014.2753

38. Hess AR, Seftor EA, Seftor RE, Hendrix MJ. Phosphoinositide 3-kinase regulates membrane Type 1-matrix metalloproteinase (MMP) and MMP-2 activity during melanoma cell vasculogenic mimicry. Cancer Res. 2003;63(16):4757-4762.

39. Lin JX, Xie XS, Weng XF, et al. UFM1 suppresses invasive activities of gastric cancer cells by attenuating the expres7sion of PDK1 through PI3K/AKT signaling. J Exp Clin Cancer Res. 2019;38 (1):410. doi:10.1186/s13046-019-1416-4

40. Piperigkou Z, Manou D, Karamanou K, Theocharis AD. Strategies to target matrix metalloproteinases as therapeutic approach in cancer. Methods Mol Biol. 2018;1731:325-348.

41. Radisky ES, Radisky DC. Matrix metalloproteinase-induced epithelial-mesenchymal transition in breast cancer. J Mammary Gland Biol Neoplasia. 2010;15(2):201-212. doi:10.1007/s10911010-9177-x
OncoTargets and Therapy

\section{Publish your work in this journal}

OncoTargets and Therapy is an international, peer-reviewed, open access journal focusing on the pathological basis of all cancers, potential targets for therapy and treatment protocols employed to improve the management of cancer patients. The journal also focuses on the impact of management programs and new therapeutic

\section{Dovepress}

agents and protocols on patient perspectives such as quality of life adherence and satisfaction. The manuscript management system is completely online and includes a very quick and fair peer-review system, which is all easy to use. Visit http://www.dovepress.com/ testimonials.php to read real quotes from published authors. 